Nicole Wendt, Jörg Tittelbach, Marc-Oliver Grimm, Cornelia Scheungraber, Bettina Löffler, Michael Baier and Matthias Karrasch*

\title{
Prospective evaluation study on the benefit of the simultaneous detection of seven sexually transmitted pathogens for the clinical management of patients suffering from sexually transmitted diseases
}

https://doi.org/10.1515/labmed-2018-0322

Received March 1, 2018; accepted September 10, 2018; previously published online November 29, 2018

\section{Abstract}

Background: Sexually transmitted infections (STIs) represent a growing relevant public health problem. Early and accurate STI diagnosis is capable of preventing the spread and severe complications of curable STIs through pathogen adapted antibiotic treatment regimens. Limitations of current STI diagnostic tools are the lack of simultaneous pathogen detection and result quantification.

Methods: A prospective analysis of clinical specimens $(\mathrm{n}=100$, from 82 symptomatic patients) from different sites of infections was performed. All samples were processed with multiplex real-time polymerase chain reaction (PCR) assay Anyplex ${ }^{\mathrm{TM}}$ II STI-7, using thermal cycler CFX96 $^{\mathrm{TM}}$. The Anyplex ${ }^{\mathrm{TM}}$ II STI-7 detection assay covers Chlamydia trachomatis (CT), Neisseria gonorrhoeae (NG), Mycoplasma genitalium (MG), Mycoplasma hominis (MH), Ureaplasma parvum (UP), Ureaplasma urealyticum (UU) and Trichomonas vaginalis (TV). STI-7 results were compared to standard methods and transferred to the attending physician for treatment adjustment.

Results: Compared to performed standard method results the assay achieved sensitivities ranging from $90.9 \%$ to

*Correspondence: Matthias Karrasch, MD, Institute of Clinical Chemistry and Laboratory Medicine, Jena University Hospital, Jena, Germany; and Institute of Medical Microbiology, Jena University Hospital, Jena, Germany, Phone: +4936419325073 ,

E-Mail: matthias.karrasch@med.uni-jena.de

Nicole Wendt, Bettina Löffler and Michael Baier: Institute of Medical Microbiology, Jena University Hospital, Jena, Germany

Jörg Tittelbach: Department of Dermatology, Jena University Hospital, Jena, Germany

Marc-Oliver Grimm: Department of Urology, Jena University Hospital, Jena, Germany

Cornelia Scheungraber: Department of Gynecology, Jena University Hospital, Jena, Germany
$100 \%$, respectively, and specificities of $100 \%$, with negative predictive values (NPV) ranging from $98.5 \%$ to $100 \%$, respectively, and positive predictive values (PPV) of $100 \%$. The Anyplex ${ }^{\mathrm{TM}}$ II STI-7 detection assay measured a Cohen's kappa of 1.00 for UU/UP, MH, and MG and a Cohen's $\kappa$ agreement of 0.95 and 0.96 for NG and CT, respectively. Conclusions: The Anyplex ${ }^{\mathrm{TM}}$ II STI-7 assay can easily be introduced into the microbiological laboratory work flow due to its short hands-on-time and PCR mutiplexity. The simultaneous detection of seven STI pathogens provides a comprehensive profile for each patient, enabling clinicians to decide on best treatment options, decreasing antibiotic misuse and infection spreading risk. The semiquantitative results enables clinicians to gain a complete package of diagnostic information including disease diagnosis, disease degree severity and treatment monitoring, although. Further clinical studies on this topic are needed.

Keywords: antibiotic stewardship; multiplex real-time PCR; sexually transmitted diseases (STD).

\section{Summary}

The clinical evaluation of a new culture-independent diagnostic tool for the fast and simultaneous detection of seven sexually transmitted pathogens in 82 symptomatic patients showed good sensitivity/specificity in pathogen detection compared to standard methods. In addition, insight could be gained towards co-infection rates, resulting pathogenadapted antibiotic treatment option and potential treatment cost reduction for each individual patient.

\section{Introduction}

Sexually transmitted infections (STIs) are a growing health problem worldwide [1]. Due to the rising 
incidence and developing antibiotic resistance of STI pathogens, health systems get confronted with increasing long-term complications and costs [2]. Four of the most prevalent infections, next to HPV, HSV-2, HIV and HBV, are currently curable: chlamydia, gonorrhea, syphilis and trichomoniasis [1]. Depending on the causative pathogen, STIs show unspecific symptoms, such as dysuria, urethral or vaginal discharge, pelvic pain and acyclic bleeding, but have serious reproductive health consequences beyond the immediate impact of the infection itself [3]. Symptoms of Mycoplasma and Ureaplasma infection are mild, but a chronic colonization leads to latent disturbed natural microbial flora and might increase the risk of acquisition of other pathogens [4]. Further they are associated with pelvic inflammatory disease (PID), which might lead to infertility $[5,6]$, and intra amnionic and postpartum infections. Different STI pathogens may cause similar symptoms, thus the distinction between them is often challenging. Multiple infections with different STI pathogens are increasing due to the high susceptibility in certain population groups, especially in men having sex with men (MSM) [7] and sex workers [8]. Current diagnostic STI screening methods are mainly single pathogen tests. Thus, multiple tests need to be performed for pathogen-adapted antibiotic therapy. Nucleic acid amplification tests (NAATs) provide rapid and accurate results with high sensitivity and specificity and became more important in diagnosing STIs over the past years [9]. Multiplex real-time polymerase chain reaction (PCR) tests are suitable for STI diagnosis due to the variety of microorganisms causing similar clinical conditions [10]. Multiplex PCR panels might be provided to clarify special medical issues, like bacterial vaginosis [11] or STI symptoms [12]. The Anyplex ${ }^{\mathrm{TM}}$ II STI-7 (Seegene, Seoul, Korea) detection assay was evaluated and compared to standard methods, including other NAATs [13]. Clinical parameters were calculated for Chlamydia trachomatis (CT), Neisseria gonorrhoeae (NG) and Trichomonas vaginalis (TV) [14]. The evaluation should be expanded by Myсoplasma hominis (MH), Mycoplasma genitalium (MG) and Ureaplasma urealyticum (UU), and Ureaplasma parvum (UP) to evaluate epidemic level with common co-infections and get in the position to assess sequelae $[15,16]$. This study investigated the diagnostic performance of a multiplex real-time PCR concerning time-to-result and cost-efficiency and the clinical usefulness of a comprehensive STI profile. A second evaluation objective was to compare multiplex PCR results from urine with urethral swabs [17] as a less painful specimen collection, also in patients without urethral discharge.

\section{Materials and methods}

\section{Subjects and samples}

Patients seeking sexually transmitted disease (STD) testing and treatment at urological, dermatological and gynecological departments of Jena University Hospital from January 2016 to December 2016 were included. Urine samples, urethral, endocervical, rectal and oral swab samples were collected based on medical history and clinical symptoms. Some of the subjects had multiple samples collected, thus 100 samples from 82 symptomatic patients were included. Swab samples were placed in Remel M4RT ${ }^{\circledR}$ transport medium (Thermo Fisher Scientific Inc., Waltham, MA, USA) or eSwab ${ }^{\mathrm{TM}}$ Liquid Amies Collection and Transport System (Copan Diagnostics, Murrieta, CA, USA). Urine samples were transferred to the laboratory in sterile containers. All specimens were processed on the day of transmittal for direct standard testing including DNA eluate preparation followed by real-time PCR and subsequent freezing.

\section{Ethical considerations}

The Ethical Review Committee of Jena University Hospital approved the study in May 2015.

\section{Standard clinical testing}

All specimens were sent in with selected test requests for single STI pathogens, according to symptoms and clinical situation. Standard tests for CT, NG and TV included real-time PCR or Giemsa staining and were performed in the microbiology laboratory of Jena University Hospital. Samples investigated for UU and MH by bacteriologic culture were sent to the laboratory of Technical University Dresden. To increase the number of comparable standard testing results for MG, MH and UP/UU, PCR and enzymelinked immunosorbent assay (ELISA) reverse hybridization were used.

\section{Chlamydia trachomatis}

For requested CT standard testing DNA extraction was performed using the Cobas AmpliPrep Instrument with TNAI CS4 Reagent (Roche, Mannheim, Germany) followed by real-time PCR using the Cobas TaqMan with TaqMan 
CT Test v2.0 Kit (Roche Deutschland Holding GmbH, Mannheim, Germany). The Cobas AmpliPrep instrument was loaded with TNAI CS4 reagent rack, reaction mixture and $1 \mathrm{~mL}$ of untreated samples for DNA extraction according to manufacturers protocol. DNA amplification and CT analyzing was accomplished by Cobas TaqMan Thermocycler with $50 \mu \mathrm{L}$ of prepared Cobas TaqMan CT Test v2.0 MM and $50 \mu \mathrm{L}$ of DNA eluate.

\section{Neisseria gonorrhoeae}

For NG standard testing DNA extraction was performed using EZ1 Advanced XL with EZ1 Virus Mini Kit v2.0. (Qiagen, Hilden, Germany) followed by real-time PCR using LightCycler® 2.0 System (Roche, Basel, Switzerland) with GeneProof NG PCR Kit (GeneProof a.s., Vídenská, Czech Republic). The transport media liquid of swab samples was used for purification of bacterial DNA. Urine was centrifuged at $18,000 \times \mathrm{g}$ for $15 \mathrm{~min}$, the supernatant, except for $300 \mu \mathrm{L}$, was discarded. The pellet was resuspended in the remaining liquid. Following, $50 \mu \mathrm{L}$ of tissue lysis buffer (ATL) were added to $150 \mu \mathrm{L}$ of the concentrated urine sample and used for DNA extraction. The DNA was extracted from the specimens (swab liquid, pretreated urine) using the EZ1 Virus Mini Kit v2.0 according to manufacturers protocol including the use of internal control (IC). Bacterial DNA was purified in one step following the automated EZ1 Virus procedure. Bacterial DNA was eluted in a volume of $60 \mu \mathrm{L}$ and amplified using the GeneProof NG PCR Kit according manufacturers protocol. For the real-time PCR using the LightCycler ${ }^{\circledR} 2.0$ System, $15 \mu \mathrm{L}$ of a ready-to-use Mastermix (MM) and $5 \mu \mathrm{L}$ of the DNA eluate were placed in capillaries. Remaining eluate was stored at $-80{ }^{\circ} \mathrm{C}$ for further experiments using multiplex real-time PCR with Anyplex ${ }^{\mathrm{TM}}$ II STD-7 detection assay.

\section{Mycoplasma hominis, Mycoplasma genitalium, Ureaplasma urealyticum and Ureaplasma parvum}

Of the 100 samples, results from 81 samples were compared to an established gold standard (ELISA) for $\mathrm{MH}$, MG and UU/UP. In method comparison. DNA extraction was performed as described with NG followed by PCR and ELISA reverse hybridization. DNA was amplified using the eppendorf Mastercycler personal (eppendorf AG, Hamburg, Germany) with the hyplex ${ }^{\circledR}$ protocol (amplex Biosystems, Gießen, Germany). Subsequent analysis (ELISA) was performed using the hyplex ${ }^{\circledR}$ STD ID panel according to manufacturers protocol.

\section{Trichomonas vaginalis}

For requested TV standard testing swab samples were dispensed onto an object slide, air-dried and afterwards fixed in methanol for 3-5 min. The slide was purged with $\mathrm{H}_{2} \mathrm{O}$, Giemsa stained for $30 \mathrm{~min}$, purged and air-dried. Slides were investigated for TV protozoon and recorded as positive when at least one protozoon was found.

\section{Multiplex real-time PCR testing}

DNA extraction of samples was performed as described with NG using the EZ1 Advanced XL with EZ1 Virus Mini Kit v2.0 (Qiagen), according to manufacturers instructions. Elution fractions of extracted DNA were stored at $-80{ }^{\circ} \mathrm{C}$ and used for weekly STI-7 testing. For each sample reaction $5 \mu \mathrm{L}$ were used and mixed with $15 \mu \mathrm{L}$ of MM and $1 \mu \mathrm{L}$ of IC. Additionally, negative (RNase-free water) and positive controls were set up as described before. Test tubes were centrifuged at $13,000 \times \mathrm{g}$ for $3 \mathrm{~min}$ and put into the CFX96 ${ }^{\mathrm{TM}}$ Thermal Cycler (Bio-Rad Laboratories, Hercules, CA, USA). According to STI-7 protocol real-time PCR and fluorescent analyzing were performed. Bio-Rad software presented semi-quantitative results such as negative and three levels of positive, according to in which cycle target DNA was detected by fluorescent analysis. Anyplex $^{\text {TM }}$ II STI-7 semi-quantitative results contained three levels of positivity, according to the appearance of bacterial DNA during amplification procedure - “+++” first cyclic Catcher Melting Temperature Analysis (CMTA) point, "++" second, "+" third.

\section{Analysis of results and statistical comparisons}

The specimens were tested with diagnostic standard for those pathogens clinicians requested. Additional positive results detected by Anyplex ${ }^{\mathrm{TM}}$ II STI-7 were considered missed by the clinician. The sensitivity, specificity, positive predictive value (PPV), negative predictive value (NPV), and kappa-index were calculated for the pathogens tested in parallel. Results from UP and UU were not analyzed individually as the used methods, such as Amplex PCR and hybridization or bacterial culture, were not able to distinguish between these pathogens. SPSS Statistics version 24.0 was used to determine quality criteria of the evaluated diagnostic method and to measure concordance between standard testing and multiplex real-time PCR. 


\section{Results}

\section{Clinical incidence of seven STI pathogens in 82 subjects}

The distribution of microorganisms in the clinical samples is shown in Table 1. The total group of 82 patients consisted of 43 patients who had negative test results, 25 patients who were positive for a single pathogen, and 14 patients who were positive for multiple microorganisms (2-4 pathogens). From all negative patients 31 were male and 13 were female patients. Regarding patients who were single microorganism positive, 21 were male and four were female patients. Approximately $80 \%$ of STIs with multiple pathogens occurred in women. The most prevalent pathogens were UP and CT with $19 \%$ and $18 \%$, respectively. The most frequently detected pathogen in female patients was UP $(n=13)$, whereas NG was most frequent in male patients $(n=10)$. Among the 100 tested samples, 42 were positive for at least one pathogen. Of the positive samples 15 (36\%) showed multiple pathogen infections. Double infection was detected in eight, triple infection in four, and fourfold infection in three samples. Constellations of STI single and multiple infections are shown in Table 2. The most prevalent microorganisms in multiple infections were UP, CT and UU, with 67\%, 60\%, and 53\%, respectively.

\section{Performance of the real-time multiplex PCR}

Anyplex $^{\mathrm{TM}}$ II STI-7 detected CT in 14, NG in 12, UP in 16, MG in three, MH in eight, UU in 11 and TV in one of the analyzed samples. The panel missed one CT (vaginal swab) and one NG (urine) infection compared to standard test results. The Anyplex ${ }^{\mathrm{TM}}$ II STI-7 panel identified 11 additional mono-infections and 15 additional multiple infections in samples not tested with standard methods (Table 3). The individually counted pathogen infections summed up to 67 , of these 40 were detected only by using the STI-7 panel, because clinicians did not request standard testing for the causing pathogens (Table 4). Compared to performed standard method results and the additionally performed hyplex PCR and ELISA analysis, the assay achieved sensitivities ranging from $90.9 \%$ to $100 \%$, and specificities of $100 \%$, with NPV ranging from $98.5 \%$ to $100 \%$, and PPV of $100 \%$. Urine and urethral swab samples of eight patients were tested in parallel. Five of those patients had all negative results in both specimens. Two were detected positive for UP in urethral swab sample, but negative in urine. In one case urine was detected positive for NG only

Table 1: Distribution of pathogen infections in the clinical samples.

\begin{tabular}{|c|c|c|c|c|c|}
\hline \multirow{2}{*}{$\begin{array}{l}\text { Organism } \\
\text { Sample positive }\end{array}$} & \multirow{2}{*}{$\begin{array}{r}\text { Total no. } \\
42\end{array}$} & \multicolumn{2}{|c|}{$\begin{array}{r}\text { Female samples }(n=33) \\
\text { No. positive, \% }\end{array}$} & \multicolumn{2}{|c|}{$\begin{array}{r}\text { Male samples }(n=67) \\
\text { No. positive, \% }\end{array}$} \\
\hline & & 16 & 48.5 & 26 & 38.8 \\
\hline Chlamydia trachomatis & 15 & 7 & 21.2 & 8 & 11.9 \\
\hline Neisseria gonorrhoeae & 13 & 3 & 9.1 & 10 & 14.9 \\
\hline Mycoplasma genitalium & 3 & 1 & 3.0 & 2 & 3.0 \\
\hline Mycoplasma hominis & 8 & 6 & 18.2 & 2 & 3.0 \\
\hline Ureaplasma parvum & 16 & 13 & 39.4 & 3 & 4.5 \\
\hline Ureaplasma urealyticum & 11 & 4 & 12.1 & 7 & 10.4 \\
\hline Trichomonas vaginalis & 1 & 0 & 0.0 & 1 & 1.5 \\
\hline All negative results & 58 & 17 & 51.5 & 41 & 61.2 \\
\hline Single migroorganism detected & 27 & 5 & 15.2 & 22 & 32.8 \\
\hline Multiple microorganisms detected & 15 & 11 & 33.3 & 4 & 6.0 \\
\hline
\end{tabular}

Organism distribution exceeds $100 \%$ because multiple microorganism positivity was counted individually.

Table 2: Set-up of microorganisms in the positive samples.

\begin{tabular}{lrl}
\hline Infection & Total no. & Pathogens \\
\hline Single & 27 & $8 \times \mathrm{NG} ; 6 \times \mathrm{UP} ; 6 \times \mathrm{CT} ; 3 \times \mathrm{UU} ; 2 \times \mathrm{MG} ; 2 \times \mathrm{MH}$ \\
Double & 8 & $2 \times \mathrm{UU}+\mathrm{CT} ; 2 \times \mathrm{UP}+\mathrm{CT} ; \mathrm{UU}+\mathrm{UP} ; \mathrm{UP}+\mathrm{NG} ; \mathrm{UU}+\mathrm{NG} ; \mathrm{UP}+\mathrm{MH}$ \\
Triple & 4 & $2 \times \mathrm{UP}+\mathrm{MH}+\mathrm{CT} ; \mathrm{UU}+\mathrm{NG}+\mathrm{CT} ; \mathrm{UU}+\mathrm{UP}+\mathrm{MH}$ \\
Fourfold & 3 & $\mathrm{UP}+\mathrm{MG}+\mathrm{MH}+\mathrm{CT} ; \mathrm{UU}+\mathrm{UP}+\mathrm{NG}+\mathrm{CT} ; \mathrm{UU}+\mathrm{MH}+\mathrm{NG}+\mathrm{TV}$ \\
\hline
\end{tabular}


Table 3: Pathogen infections additionally detected by Anyplex ${ }^{\mathrm{TM}}$ II STI-7 in samples without clinicians request for standard testing.

\begin{tabular}{|c|c|c|c|c|c|c|}
\hline \multirow[t]{2}{*}{ Organism positive } & \multicolumn{2}{|r|}{ Total } & \multicolumn{2}{|c|}{ Female samples } & \multicolumn{2}{|c|}{ Male samples } \\
\hline & Infections & Pos. S & Infections & Pos. S & Infections & Pos. S \\
\hline Any pathogen & 40 & 26 & 25 & 15 & 15 & 11 \\
\hline Single pathogen & 11 & 11 & 4 & 4 & 7 & 7 \\
\hline Multiple pathogens & 29 & 15 & 21 & 11 & 8 & 4 \\
\hline
\end{tabular}

The total amount and distribution of single and multiple pathogen infections in positive female and male samples (S) are shown.

Table 4: Additionally detected pathogen infections by Anyplex ${ }^{\mathrm{TM}}$ II STI-7 and share of total infections.

\begin{tabular}{llrr}
\hline Organism positive & $\begin{array}{l}\text { Share of total } \\
\text { infections }\end{array}$ & $\begin{array}{r}\text { Female } \\
\text { samples }\end{array}$ & $\begin{array}{r}\text { Male } \\
\text { samples }\end{array}$ \\
\hline Chlamydia trachomatis & $1 / 15 \mathrm{Inf}$. & 0 & 1 \\
Neisseria gonorrhoeae & $2 / 13 \mathrm{Inf}$. & 1 & 1 \\
Mycoplasma genitalium & $3 / 3 \mathrm{Inf}$. & 1 & 2 \\
Mycoplasma hominis & $8 / 8 \mathrm{Inf}$. & 6 & 2 \\
Ureaplasma parvum & $16 / 16 \mathrm{Inf}$. & 13 & 3 \\
Ureaplasma urealyticum & $9 / 11 \mathrm{Inf}$. & 4 & 5 \\
Trichomonas vaginalis & $1 / 1 \mathrm{Inf}$. & 0 & 1 \\
\hline
\end{tabular}

by the standard method, while the swab sample was negative in both tests, standard and multiplex real time PCR. The total of 65 pathogen detections comprised $17 \times$ at first CMTA, $41 \times$ at second CMTA and $7 \times$ at third CMTA. Single and multiple infections were detected at any of the three points of CMTA. NG set up the largest part (7/18 infections) of first CMTA point positivity. UU, UP and CT were mostly detected by second CMTA point with 12, nine, and eight of 42 infections, respectively.

\section{Clinical sensitivity, specificity, PPV and NPV}

The sensitivity, specificity, PPV and NPV for CT, NG, MH, MG, UU/UP and UU using the gold standard method. Anyplex $^{\text {TM }}$ II STI-7 had $100 \%$ sensitivity and $100 \%$ specificity for $\mathrm{MH}, \mathrm{MG}$ and UU/UP within the 81 standard tested samples using Amplex PCR and hybridization. Twenty-six samples were sent to Technical University of Dresden for culture testing. The results showed a sensitivity of $66.7 \%$ and specificity of $95.7 \%$ for UU compared to bacteriologic culture. All 26 specimens were detected negative for $\mathrm{MH}$, by culture and by Anyplex ${ }^{\mathrm{TM}}$ II STI-7. From the 84 samples tested with Cobas TaqMan, one vaginal swab sample was discordant for the detection of CT. It showed a sensitivity of $92.9 \%$ and specificity of $100 \%$ for CT. It missed one NG infection (urine) in 75 samples tested in parallel, leading to sensitivity of
$90.9 \%$ and specificity of $100 \%$ for NG. In the 28 samples tested for TV, neither the staining nor Anyplex ${ }^{\mathrm{TM}}$ II STI-7 detected an infection, representing an agreement of $100 \%$ between the two tests. The Anyplex ${ }^{\mathrm{TM}}$ II STI-7 detection assay measured a Cohen's $\kappa$ of 1.00 for UU/UP, $\mathrm{MH}$ and MG, and a Cohen's $\kappa$ agreement for NG and CT of 0.95 and 0.96 , respectively.

\section{Discussion}

\section{Comparison to standard tests}

The Anyplex ${ }^{\mathrm{TM}}$ II STI-7 detection assay showed good concordance to the performed standard real-time PCR for CT and NG detection. Bacteriologic culture as a standard method for $\mathrm{MH}$ and UU testing may detect living microorganisms for antibiotic susceptibility testing, but is not very reliable due to the interference-prone transport and detection procedure. To cultivate sensitive pathogens, rapid and accurate processing of the specimens with living microorganisms is needed. MH and UU culture is timeconsuming, expensive and difficult to perform. Additionally, culture is not very specific for UU, because UU and UP cultures look alike. All MH infections and the majority of Ureaplasma infections were detected in samples with no request for the standard tests. These infections were detected by the STI-7 panel and approved by the additional reference Amplex PCR and hybridization. This led to a high rate of infections that would have been missed by the clinician due to inconclusive clinical symptoms. Long-established diagnostic methods, such as culture and serology are not suitable for mollicutes, because of the criteria set out above. Whereas the panel showed high agreement using the Amplex PCR and hybridization as reference method, though the reference method was not able to differentiate between UU and UP. It is a major advantage of the STI-7 panel to distinguish between these two species, because UP are considered commensals without or at least with controversial pathogenicity $[5,6]$. 
In this study, only symptomatic patients were enrolled. Especially young patients showed persistent clinical signs, thus pathogenicity seems to be relevant - nevertheless treatment is necessary in patients showing signs of inflammation and infection [18]. One further advantage of multiplex real-time PCR testing in comparison to standard diagnostic methods is the improved hands-on-time and total time to results for detecting multiple pathogens (Table 2). Therefore multiplex testing is considered a costeffective and timesaving diagnostic method in a diagnostic microbiology laboratory.

\section{Multiplex testing for STI detection}

Infection symptoms from one pathogen might be superimposed by clinical symptoms from another pathogen, or might even be similar. Multiplex assay results provide infection profiles with information about co-existing pathogens [19]. Various gold standard procedures are available in microbiological laboratories, such as Gram stain, culture, NAATs or different PCRs. In order to simplify laboratory work, the detection of multiple pathogens causing similar clinical symptoms might be combined in one device. That means lower effort and complexity of time consuming diagnostic methods to generate a comprehensive infection profile. Testing specimens from different infection sites and various sample types, such as swabs, urine, sperm, exprimate urine, leads to a wide range of diagnostic possibilities. Though STIs are showing similar clinical symptoms, different antibiotic therapy is indicated. Due to the comprehensive and specific pathogen detection, antibiotic class can be selected for successful treatment. Limitations of NAATs are discussed, such as problems with their specificity in pharyngeal and anal swab sample diagnostics. The reason for this are false positive results of non-pathogenic Neisseria [20]. High specificity for NG of the reference test and Anyplex ${ }^{\mathrm{TM}}$ II STI-7 detection assay has been approved before [21]. The detection without cultivation of living microorganisms lacks consecutive antibiograms. Developing resistance in STI pathogens, especially for NG and mollicutes, should be considered in case of low response to antibiotic treatment $[22,23]$.

\section{Pathogens included in detection panels}

Approaches have been made for comprehensive STI tests covering up to 18 pathogens $[24,25]$. The pathogens included in diagnostic panels should have similar infection symptoms, so that clinical assessment is in inferior position to the comprehensive test method. In addition pathogen selection should impact on therapy or observation options to entail best treatment. Therefore, multiplex PCR testing is reasonable in analyzing the cause of unspecific symptoms and should include various microorganisms causing similar clinical situations. The detection method might not be indicated for viral infections without subsequent therapy option and high infestation, such as HSV-2. It is less indicated for STIs with typical symptoms and clinical course with a highly established and sensitive gold standard method, for example, syphilis considering clinically apparent stage I and monitoring serology in the following stages [20]. Also regional differences in pathogen prevalence should be considered, for example, TV in this study cohort. The extent of the analysis in terms of time and size, considering pathogen selection, should be adjusted to regional, clinical and therapeutic situation.

\section{Semi-quantitative results}

To limit the use of antibiotics to the cases indicated [26], cutoffs for detecting infection and questionable correlation of germ load and severity should be considered. Quantification is one of the most important features of real-time PCR. To find out whether the semi-quantitative results correlate with the severity of infection, its distribution was examined through single and multiple infection patterns. Control screening and therapy monitoring with real-time PCR and semi-quantitative results is conceivable, though further analysis concerning correlation of pathogen load and infection severity is needed. Due to detecting DNA of both living and dead microorganisms, a control test should be performed at least 8 weeks after antibiotic treatment. During that period of time, reinfection and incubation with other pathogens are possible [27].

\section{Conclusions}

The Anyplex ${ }^{\mathrm{TM}}$ II STI-7 assay can easily be introduced into the microbiological laboratory workflow due to its short hands-on-time and PCR multiplexity. The simultaneous detection of STI pathogens and semi-quantitation might enable clinicians to better diagnose STD disease severity, treatment and monitoring. This assay might thus be able to serve as an antibiotic stewardship program and an 
early STD infection control tool, but further clinical prospective studies are needed. Different sample types can be measured using the Anyplex ${ }^{\mathrm{TM}}$ II STI-7 multiplex real-time PCR, but whether urine is as conclusive as urethral swab samples in infection diagnosing could not be determined in this study.

Acknowledgments: The authors would like to thank Beate Haschke, Institute of Medical Microbiology, Jena University Hospital for valuable technical assistance with specimen handling. Furthermore, the authors would like to thank Sung Zun Park, MBA (Seegene Germany, Eschborn, Germany), Younjung Shim, BSc and Soung Youl Kim, PhD (both Seegene Inc., Seoul, South Korea) for supporting this trial by providing Seegene Anyplex ${ }^{\mathrm{TM}}$ II STI-7 hardware and software for free during the evaluation period.

Author contributions: All the authors have accepted responsibility for the entire content of this submitted manuscript and approved submission.

Research funding: The Anyplex ${ }^{\mathrm{TM}}$ II STI-7 system was provded for free during the trial period from Seegene Inc, Seoul, Korea. MK received a travel grant to present preliminary study results at the European Conference for Clinical Microbiology and Infectious Diseases (ECCMID) 2017 in Vienna, Austria.

Employment or leadership: None declared.

Honorarium: None declared.

Competing interests: The funding organization(s) played no role in the study design; in the collection, analysis, and interpretation of data; in the writing of the report; or in the decision to submit the report for publication.

\section{References}

1. World Health Organization. Media centre: sexually transmitted infections (STIs) - fact sheets 2017. Accessed: 26 Apr 2017.

2. Centers for Disease Control and Prevention. CDC fact sheet: incidence, prevalence, and cost of sexually transmitted infections in the United States 2013. Available at: https://www.cdc.gov/std/ stats/sti-estimates-fact-sheet-feb-2013.pdf. Accessed: $30 \mathrm{Apr}$ 2017.

3. Schuchardt L, Rupp J. Chlamydia trachomatis as the cause of infectious infertility: acute, repetitive or persistent long-term infection? Curr Top Microbiol Immunol 2016;412:159-82.

4. Paulsen LK, Dahl ML, Skaare D, Grude N. Prevalence of M. genitalium and $\mathrm{U}$. urealyticum in urine tested for C. trachomatis. Tidsskr Nor Laegeforen 2016;136:121-5.

5. Sleha R, Bostikova V, Hampl R, Salavec M, Halada P, Štěpán $M$, et al. Prevalence of Mycoplasma hominis and Ureaplasma urealyticum in women undergoing an initial infertility evaluation. Epidemiol Mikrobiol Imunol 2016;65:232-7.
6. Cox C, Saxena N, Watt AP, Gannon C, McKenna JP, Fairley DJ, et al. The common vaginal commensal bacterium Ureaplasma parvum is associated with chorioamnionitis in extreme preterm labor. J Matern Fetal Neonatal Med 2016;29:3646-51.

7. Centers for Disease Control and Prevention. Sexually transmitted diseases surveillance: STDs in men who have sex with men 2014. Available at: https://http://www.cdc.gov/std/stats14/ msm.htm. Accessed: 4 Aug 2017.

8. Centers for Disease Control and Prevention. HIV risk among persons who exchange sex for money or nonmonetary items 2016. Available at: https://www.cdc.gov/hiv/group/sexworkers.html. Accessed: 4 Aug 2017.

9. Skulska E, Mlynarczyk-Bonikowska B, Walter de Walthoffen S, Mtynarczyk G, Malejczyk M, Majewski S. The comparison of realtime PCR and direct immunofluorescence in laboratory diagnostics of chlamydiosis in patients of Department of Dermatology and Venereology Medical University of Warsaw. Medycyna doswiadczalna i mikrobiologia 2015;67:173-80.

10. Yu B, An Y, Xu G, Shan H. Detection of Chlamydia trachomatis and Neisseria gonorrhoeae based on cross-priming amplification. Lett Appl Microbiol 2016;62:399-403.

11. Hilbert DW, Smith WL, Chadwick SG, Toner G, Mordechai E, Adelson ME, et al. Development and validation of a highly accurate quantitative real-time PCR assay for diagnosis of bacterial vaginosis. J Clin Microbiol 2016;54:1017-24.

12. Kriesel JD, Bhatia AS, Barrus C, Vaughn M, Gardner J, Crisp RJ. Multiplex PCR testing for nine different sexually transmitted infections. Int J STD AIDS 2016;27:1275-82.

13. Choe HS, Lee DS, Lee SJ, Hong SH, Park DC, Lee MK, et al. Performance of Anyplex (TM) II multiplex real-time PCR for the diagnosis of seven sexually transmitted infections: comparison with currently available methods. Int J Infect Dis 2013;17:E1134-40.

14. Fernandez G, Martro E, Gonzalez V, Saludes V, Bascuñana E, Marcó C, et al. Usefulness of a novel multiplex real-time PCR assay for the diagnosis of sexually-transmitted infections. Enferm Infec Micr Cl 2016;34:471-6.

15. Ikonomidis A, Venetis C, Georgantzis D, Giaslakiotis V, Kolovos V, Efstathiou K, et al. Prevalence of Chlamydia trachomatis, Ureaplasma spp., Mycoplasma genitalium and Mycoplasma hominis among outpatients in central Greece: absence of tetracycline resistance gene tet(M) over a 4-year period study. New Microbes New Infect 2016;9:8-10.

16. Lee MY, Kim MH, Lee WI, Kang SY, Jeon Y. Prevalence and antibiotic susceptibility of Mycoplasma hominis and Ureaplasma urealyticum in pregnant women. Yonsei Med J 2016;57:1271-5.

17. Gueye SB, Diop-Ndiaye H, Gningue A, Ndiaye O, Mbengue AS, Gaye-Diallo A, et al. Performance of the Abbott Real Time CT/ NG assay in urines and cervico-vaginal samples from Senegal. J Infect Dev Countr 2014;8:898-903.

18. Zeng XY, Xin N, Tong XN, Wang JY, Liu ZW. Prevalence and antibiotic susceptibility of Ureaplasma urealyticum and Mycoplasma hominis in Xi'an, China. Eur J Clin Microbiol Infect Dis 2016;35:1941-7.

19. Kim Y, Kim J, Lee KA. Prevalence of sexually transmitted infections among healthy Korean women: implications of multiplex PCR pathogen detection on antibiotic therapy. J Infect Chemother 2014;20:74-6.

20. Mlynarczyk-Bonikowska B, Skulska E, Malejczyk M. Nucleic acid amplification methods in laboratory diagnostics of selected sexually transmitted diseases. Postep Mikrobiol 2015;54:407-14. 21. Seegene Inc. Anyplex II STI-7 Technical Manual. 2015. 
22. Wang QY, Li RH, Zheng LQ, Shang XH. Prevalence and antimicrobial susceptibility of Ureaplasma urealyticum and Mycoplasma hominis in female outpatients, 2009-2013. J Microbiol Immunol Infect 2016;49:359-62.

23. Skiljevic D, Mirkov D, Vukicevic J. Prevalence and antibiotic susceptibility of Mycoplasma hominis and Ureaplasma urealyticum in genital samples collected over 6 years at a Serbian university hospital. Indian J Dermatol Venereol Leprol 2016;82:37-41.

24. Schmitt M, Depuydt C, Stalpaert M, Pawlita M. Bead-based multiplex sexually transmitted infection profiling. I Infect 2014;69:123-33.
25. Kweon OJ, Choi JH, Song UH, Park AJ. Performance evaluation of a DNA chip assay in the identification of major genitourinary pathogens. J Microbiol Methods 2015;109:117-22.

26. Kim Y, Kim J, Lee KA. Analytical performance of multiplex real-time PCR for six sexually transmitted pathogens. Clin Lab 2015;61:1749-54.

27. Bissessor M, Whiley DM, Fairley CK, Bradshaw CS, Lee DM, Snow AS, et al. Persistence of Neisseria gonorrhoeae DNA following treatment for pharyngeal and rectal gonorrhea is influenced by antibiotic susceptibility and reinfection. Clin Infect Dis 2015;60:557-63. 